\title{
Uso das Dissertações Desenvolvidas nos Programas de Pós-Graduação em Ciências Contábeis na Sustentação e Desenvolvimento de Artigos Científicos Publicados em Eventos da Área ${ }^{1}$
}

\author{
Luzivalda Guedes Damascena ${ }^{2}$ \\ Karla Roberta Castro Pinheiro ${ }^{3}$ \\ Paulo Roberto Nóbrega Cavalcante ${ }^{4}$
}

\section{RESUMO}

O presente trabalho objetiva identificar o perfil da utilização das dissertações dos PPGCCs na sustentação e no desenvolvimento de artigos científicos publicados em eventos na área contábil, no Brasil. A pesquisa é do tipo descritiva, por apresentar as dissertações utilizadas no construto das publicações científicas da área contábil; é bibliográfica e documental, e a técnica de pesquisa utilizada é a análise de conteúdo. Analisaram-se os artigos dos eventos ANPCont, USP e EnANPAD, nos períodos de 2007 a 2009, totalizando 1.292 artigos verificados, sendo que, desses, 480 utilizam dissertações para a sustentação e o desenvolvimento de artigos científicos da área. Os resultados revelam que as dissertações do PPGCC da USP são as mais utilizadas no processo de desenvolvimento de artigos na área contábil. Constata-se que Gestão de Operações e Logística é um campo de estudo a ser explorado pelos pesquisadores, devido à possível inexistência de dissertações em contabilidade sobre essa temática. Considera-se que esta pesquisa evidencia, entre outras conclusões, potenciais campos de estudos, mas sugere-se que novas pesquisas continuem essa observância em áreas determinadas, ou com a utilização de periódicos, por exemplo, para corroborar, ou não, os resultados aqui obtidos.

Palavras-chave: Dissertações dos PPGCCs. Artigos científicos em contabilidade. Eventos na área contábil.

\begin{abstract}
This study aims to identify the usage profile of the dissertations of the Masters Programs in Accounting (MPA) support and development of scientific articles published in events in the accounting area, in Brazil. The research is descriptive, for presenting the lectures used in the construct of the area's scientific book, it is literature and documentary, and research technique used is content analysis. We analyzed the articles of events ANPCont, USP and EnANPAD in the periods 2007 to 2009, totaling 1,292 items checked, and, among these, 480 dissertations used for support and development of scientific papers in the area. The results show that the dissertations MPA of USP are the most used in the development of articles in accounting. There is a field of study to be explored by researchers due to the possible absence of dissertations in accounting on the following themes: management of operations and logistics. It is considered that this research shows, among other findings, potential fields of study, but it is suggested that further research continue this observance in certain areas, or with the use of journals, for example, to support or not, the results here obtained.
\end{abstract}

Keywords: Dissertations from PPGCCs. Papers in accounting. Events in accounting.

\footnotetext{
${ }^{1}$ Artigo publicado nos anais do XVII Congresso Brasileiro de Custos 2010, Belo Horizonte/MG. Artigo recebido em 17.11.2011. Revisado por pares em 16.02.2012 (blind review). Reformulado em 17.02.2012. Recomendado para publicação em 19.02.2012 por José Ribamar Marques de Carvalho (Editor). Publicado em 24.03.2012. Organização responsável pelo periódico: UACC/UFCG.

${ }^{2}$ Mestra em Ciências Contábeis UnB/UFPB/UFRN, e-mail: luzivaldaguedes@ gmail.com

${ }^{3}$ Mestra em Ciências Contábeis UnB/UFPB/UFRN, e-mail: karlarobertap@ hotmail.com

${ }^{4}$ Doutor em Ciências Contábeis - FEA/USP. Professor da UFPB, e-mail: prncavalcante@ ccsa.ufpb.br
}

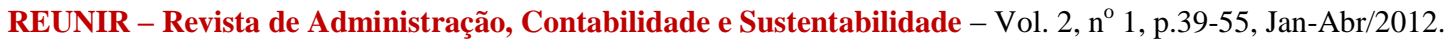




\section{INTRODUÇÃO}

O conhecimento científico é caracterizado pelo modo de conhecer e pelos instrumentos do conhecer, sendo capaz de analisar, de explicar, de desdobrar, de justificar, de induzir ou aplicar leis, de predizer com segurança eventos futuros (RUIZ, 2006). Nesse sentido, muitas produções científicas têm contribuído com as diversas ciências, entre elas a ciência contábil.

O processo de pesquisa em contabilidade percorre, por exemplo, diversos eventos ou congressos e periódicos da área, restando aos pesquisadores à geração e a interação das descobertas aos interessados por meio desses canais de comunicação. Conforme Gil (2009), os periódicos retratam pesquisas mais recentes da área, e os anais de encontros científicos constituem os locais privilegiados para apresentação de comunicação científica.

O salto da pesquisa contábil na última década, propiciado pelo surgimento e crescimento de Programas de Pós-Graduação em Ciências Contábeis (PPGCC) Stricto Sensu, tem gerado diversas discussões sobre qualidade, quantidade, desafios e perspectivas da pesquisa contábil. Lucena, Fernandes e Cavalcante (2009) mencionam que "os programas de pós-graduação externalizam as suas pretensões e apontam qual o foco do olhar dos seus pesquisadores por meio das linhas de pesquisa eleitas".

Martins (2009, p. 46) exprime, ainda, que "a criação e consolidação de cursos de pósgraduação stricto sensu são fatores fundamentais para a formação de cidadãos críticos, com habilidades e competências para intervir afirmativamente nos processos sociais e políticos locais e regionais".

Os PPGCCs, a cada triênio, são submetidos à avaliação pela Capes, entre as exigências, uma está relacionada com o que está sendo desenvolvido como pesquisas científicas na área. Isso com o intuito de estabelecer um padrão de qualidade exigido dos cursos de mestrado e doutorado. A pesquisa científica nesses programas stricto sensu se propaga através de dissertações, teses, artigos publicados em periódicos e anais de encontros científicos, dentre outros, que em geral são utilizados como fonte de pesquisa para a construção de outros trabalhos científicos.

Diante do elenco dos tipos de estudos supracitados, nota-se que as teses e as dissertações são produções importantes para a divulgação da pesquisa, em virtude da robustez do conteúdo abordado (GIL, 2009). Nesse sentido, a plataforma teórica de artigos científicos normalmente corrobora a base teórica apresentada, entre outras fontes, nas dissertações, e espera-se que o referencial usado na elaboração de artigos tenha, entre outras, validade, robustez e atualidade, a fim de contribuir com a qualidade das pesquisas.

Numa visão mais perceptiva observa-se que, dentre alguns critérios de avaliação de artigos submetidos a diversos eventos, os avaliadores têm primado por características, como as citadas anteriormente, e também pela utilização de uma literatura mais atualizada na sustentação da plataforma teórica de artigos científicos.

Conforme Santos, Lima e Martins (2009), a consulta de livros, artigos científicos, teses, jornais, dissertações, entre outros, são fontes ou materiais aproveitados e são refletidos e informados ao final do texto por meio da relação bibliográfica, referencial bibliográfico, ou simplesmente referências. Os autores ressaltam que a análise e a avaliação do levantamento bibliográfico permitem reflexões quanto aos tipos e às características das fontes utilizadas no processo de construção do conhecimento e da prática científica na elaboração de estudos.

Nesse sentido, várias pesquisas do tipo bibliométrico, por exemplo, têm buscado, de forma sistemática, analisar artigos publicados em eventos no intuito de destacar as

REUNIR - Revista de Administração, Contabilidade e Sustentabilidade - Vol. 2, nº 1, p.39-55, Jan-Abr/2012. 
abordagens, tendências e diversas características dessa produção (CHAN; MILANI FILHO; MARTINS, 2007; GOMES et al., 2008; NASCIMENTO; JUNQUEIRA; MARTINS, 2009).

No entanto, apesar de todas essas discussões, faz-se necessário evidenciar de maneira mais enfática a utilização das dissertações dos PPGCCs no processo evolutivo da produção de artigos científicos. Diante desse cenário o estudo possui o seguinte questionamento: Qual o perfil da utilização das dissertações dos PPGCCs na sustentação e no desenvolvimento de artigos científicos publicados em eventos na área contábil?

Este estudo tem por objetivo identificar o perfil da utilização das dissertações dos PPGCCs na sustentação e no desenvolvimento de artigos científicos publicados em eventos na área contábil, no Brasil. Devido à abrangência do objetivo e para alcançar melhores resultados, e, em identificar o perfil, objetiva-se especificamente:

- Verificar, nas referências dos artigos, a utilização das dissertações dos PPGCCs como fontes teóricas para o processo da pesquisa contábil no Brasil;

- Verificar a área temática do congresso analisado que utiliza mais dissertações dos PPGCCs.

- Identificar o período das dissertações citadas, no intuito de inferir se há uma utilização de material recente na produção desses artigos; e

- Conhecer os PPGCCs que mais contribuem na produção científica, relacionando as áreas temáticas desses programas e dos eventos analisados.

A pesquisa se justifica pela necessidade de se conhecer o perfil da utilização das dissertações dos PPGCCs como base teórica para produção científica de artigos em eventos mais bem conceituados, no intuito de inferir considerações a respeito da real situação desses programas no cenário atual da pesquisa contábil. Ressalta-se que o delineamento desse perfil se dá apenas na observação de dissertações, não objetivando a comparação com utilização de outras fontes de pesquisa, como livros e teses.

Assim, para melhor entendimento sobre a temática abordada, é apresentada, além da seção introdutória, uma breve revisão bibliográfica, em seguida discorre-se sobre os procedimentos metodológicos, a apresentação e as análises dos resultados, e, por fim, são realizadas as considerações finais e as sugestões para futuros estudos.

\section{REFERENCIAL TEÓRICO}

Nessa seção serão abordados assuntos inerentes à pesquisa contábil no Brasil, seguido de discussão sobre a utilização das dissertações dos PPGCCs na construção e desenvolvimento de artigos

\subsection{Pesquisa contábil no Brasil}

O tema "educação e pesquisa em contabilidade" tem sido entendido como relevante e servido de objeto de preocupação dos estudiosos, fato este que pode ser comprovado pela existência de área específica para a discussão do assunto nos principais eventos da área contábil no Brasil.

Alguns estudos, como os de Riccio, Sakata e Carastan (1999), Frezatti e Borba (2000) e Theóphilo e Iudicibus (2005), são referenciados por artigos, dissertações e teses na área de educação em contabilidade, por discorrerem e evidenciarem questões relativas à qualidade, à quantidade, aos desafios e às perspectivas da pesquisa contábil. Portanto, citam-se as

REUNIR - Revista de Administração, Contabilidade e Sustentabilidade - Vol. 2, nº 1, p.39-55, Jan-Abr/2012. 
pretensões e os resultados desses estudos e de outros, para melhor conhecimento do estado da arte acerca da temática abordada.

Riccio, Sakata e Carastan (1999) realizaram um estudo com base nas dissertações e teses existentes no período de 1962 a 1999, levando em consideração a metodologia e suas áreas temáticas. Um dos resultados indica que a temática educação na contabilidade teve um pico de produção entre 1988 e 1990, mas vem caindo progressivamente desde então.

Frezatti e Borba (2000) identificaram as principais características de tendência voltada para revistas científicas da área de contabilidade publicada na língua inglesa, e sugeriram maior agressividade no acesso a publicações em veículos internacionais.

Theóphilo e Iudicibus (2005) apresentam uma evolução favorável dos trabalhos na área de contabilidade. Mendonça Neto, Riccio e Sakata (2006) analisaram a evolução temporal da produção da pesquisa em contabilidade sob uma visão normativa e positiva no período de 1981 a 2005 nos anais do EnANPAD. Eles observaram que as pesquisas contábeis estão seguindo uma tendência baseada na pesquisa positiva.

Gomes et al. (2007) investigaram se as dissertações do PPGCC UnB/UFPB/UFRN/UFPE estavam contemplando as etapas essenciais de uma produção científica. Os autores concluíram que a maioria das dissertações do referido PPGCC contemplavam as etapas precípuas de uma produção científica, mas ressaltaram a constatação de alguns casos nos quais o questionamento da pesquisa foi omitido, ou simplesmente, não posto.

Spessato e Bezerra (2008) verificaram a utilização da abordagem positiva e normativa no desenvolvimento da pesquisa contábil nas dissertações dos PPGCCs stricto sensu da USP, da UNB e da FURB, no período de 1998 a 2007. Evidenciaram que há uma predominância da pesquisa com abordagem positiva e da linha de pesquisa em contabilidade de custos no Brasil.

Lucena, Fernandes e Cavalcante (2009), que, assim como outros autores, também fizeram menção a alguns dos estudos supracitados, buscaram investigar as linhas de pesquisas seguidas pelos PPGCCs stricto sensu, visando delinear as tendências, os desafios e as perspectivas da pesquisa. Os autores, no que se refere aos desafios no processo de pesquisa, encontraram temas, como: simulação Monte Carlo, análise fatorial, custeio híbrido, entre outros.

Identificaram, também, que as perspectivas da pesquisa para as novas dissertações giram em torno da contabilidade positiva, e que a tendência dos tratamentos dos dados segue uma tendência de modelos contábeis como aqueles que abordam os retornos anormais e o gerenciamento de resultados, e a busca pelo conhecimento da contabilidade internacional segue usando padrões, normas e comparações. Observaram que existem dois temas que começam a ser trabalhados e destacam a possibilidade de virarem um "modismo", são eles: Behavioral Accounting e Accounting Choice.

Assim, nota-se que a temática já vem sendo bastante discutida, evidenciando o estado da arte, as necessidades, as perspectivas e diversos outros detalhamentos advindos, por exemplo, de pesquisas bibliométricas, como algumas já citadas anteriormente. No entanto, há uma necessidade de se conhecer a participação das dissertações, como fontes teóricas robustas e atuais, no processo de execução de artigos, a fim de identificar algumas características e perfil dessas dissertações e suas áreas temáticas.

REUNIR - Revista de Administração, Contabilidade e Sustentabilidade - Vol. 2, nº 1, p.39-55, Jan-Abr/2012. 


\subsection{Utilização das dissertações dos PPGCCs na construção e desenvolvimento de artigos}

$\mathrm{Na}$ área acadêmica, o processo de pesquisa se encontra localizado, basicamente, no âmbito dos cursos de pós-graduação stricto sensu, os quais possuem a responsabilidade pelas pesquisas melhor estruturadas (LUCENA; CAVALCANTE; SALES, 2007). Souza (2005), ao discorrer sobre a importância de um PPGCC que estudara na ocasião, destacou, entre outras coisas, a importância do Programa para a evolução das pesquisas em contabilidade, e salientou sobre a produção de textos acadêmicos que eram desenvolvidos como sendo de boa qualidade.

Portanto, estabelece-se, mais uma vez, a prerrogativa da qualidade de estudos desenvolvidos dentro dos PPGCCs como fonte de pesquisa para produção de artigos. Diante dessa afirmação o processo de pesquisa constitui-se principalmente na produção de artigos, dando contribuições à área contábil, pois há uma grande produção de artigos ano a ano. Isso é perceptível, por exemplo, verificando a quantidade de eventos/congressos e periódicos que, por ano, aceitam centenas de trabalhos dessa natureza. Para se ter uma ideia, os eventos ANPCont, USP e EnANPAD (este último, considerando só a área contábil), juntos, aprovam cerca de 400 artigos por ano.

Em comum, esses eventos possuem área temática exclusivamente voltada a pesquisas sobre educação em contabilidade. Nessa área temática, algumas pesquisas evidenciam que as dissertações dos programas de pós-graduação ainda não possuem significativa participação na produção de artigos científicos para construção de referencial teórico.

Diehl, Souza e Alves (2008) identificaram, nas publicações sobre o método de Custeio Baseado em Atividades (ABC), que, entre as obras consultadas, somente 149 teses ou dissertações $(4,2 \%)$ foram utilizadas para sustentação e discussão teórica de artigos, onde se esperaria maior contribuição teórica, estando os mesmos essencialmente baseados no arcabouço teórico de livros textos e autores clássicos.

Ademais, outros estudos também evidenciam a pouca adoção de dissertações para construção de artigos nesse mesmo sentido (CHAN; MILANI FILHO; MARTINS, 2007; GOMES et al., 2008; NASCIMENTO; JUNQUEIRA; MARTINS, 2009). Outro fator importante a se considerar refere-se à utilização de trabalhos mais recentes, mas Nascimento, Junqueira e Martins (2009) constatam, entre outros fatores, uma alta idade média (10 anos) dos trabalhos citados em artigos, nos mesmos eventos analisados neste estudo, para a área de contabilidade gerencial.

Assim, a investigação das linhas de pesquisa seguidas pelos programas de pósgraduação, se não é suficiente para validar as pesquisas realizadas no âmbito de cada um deles, é suficiente para indicar os espaços que os pesquisadores da área contábil estão dirigindo o olhar e, até mesmo, se tal opção está de acordo com as necessidades do país (LUCENA; FERNANDES; CAVALCANTE, 2009).

\section{PROCEDIMENTOS METODOLÓGICOS}

A pesquisa é do tipo descritiva, por apresentar o perfil da utilização das dissertações no construto de artigos científicos da área contábil, bem como o período de publicação da pesquisa utilizada e a área dos eventos que faz mais uso das dissertações. É importante enfatizar que não faz parte do objeto de análise investigar a robustez teórica dos artigos, mas apenas verificar se estes utilizam dissertações. Essa informação foi construída com base nas referências de cada artigo analisado.

REUNIR - Revista de Administração, Contabilidade e Sustentabilidade - Vol. 2, nº 1, p.39-55, Jan-Abr/2012. 
O estudo é bibliográfico, por utilizar-se de materiais já elaborados, como: livros, artigos e dissertações na construção do referencial; e documental no que se refere aos dados coletados. Para o atendimento dos objetivos propostos, a técnica de pesquisa utilizada é a análise de conteúdo, pois, conforme Gil $(2009$, p. 89) a técnica da "análise de conteúdo possibilita a descrição do conteúdo manifesto e latente das comunicações", podendo ser utilizada em estudos qualitativos ou quantitativos.

O perfil foi estabelecido em função dos objetivos específicos deste estudo, ou seja, relacionando a área temática do evento e do PPGCC, o número de vezes que a dissertação foi usada etc. Assim, a pesquisa se deu a partir da catalogação e da análise das referências bibliográficas dos artigos, a fim de compor a amostra e a posterior análise dos dados, tendo como cenários da pesquisa três congressos, a saber: ANPCont, USP de Controladoria e Contabilidade e EnANPAD, nos períodos de 2007 a 2009. A escolha dos congressos ocorreu a partir da premissa de que eles se configuram como importantes e representativos veículos de disseminação da pesquisa na área contábil, corroborado o reconhecimento Qualis/Capes como eventos de alto nível, quando ainda ocorria o ranqueamento dos eventos.

Do levantamento da quantidade de trabalhos nesses eventos, foram encontrados 1.292 artigos (201 no ANPCont, 272 na USP e 819 no EnANPAD), desse total, 480 utilizam dissertações de um modo geral (foco de análise da pesquisa). Salienta-se que, no intuito de garantir maior confiabilidade das inferências deste estudo, os procedimentos adotados incluíram, inicialmente, a análise por um dos pesquisadores e posterior checagem por outro pesquisador, separadamente. Entretanto, como qualquer outro critério de classificação, está sujeito à subjetividade.

\subsection{Coleta de dados}

A pesquisa contemplou todos os artigos reunidos nos congressos supracitados, sendo a coleta de dados realizada a partir dos arquivos eletrônicos disponíveis em CD-ROM e nos sítios desses eventos. O período analisado coincide com o triênio de avaliação pela Capes para pontuar programas de pós-graduação. Assim, o resultado dessa avaliação poderá, posteriormente, ser considerado em novas inferências.

A verificação das referências dos artigos, quanto à utilização das dissertações dos PPGCCs como fontes teóricas, se deu pelos procedimentos de coleta seguintes:

a) Selecionaram-se as áreas temáticas dos congressos a serem investigadas;

b) A partir do programa Adobe Reader 9.0, utilizando a ferramenta de localização, buscou-se a palavra "dissertação", para encontrar as que faziam parte das referências do artigo;

c) Após a identificação da dissertação na referência, observaram-se as características, tais como: origem, área da dissertação e ano de sua produção/publicação;

d) As dissertações consideradas neste estudo referem-se apenas aos Programas de Mestrado em Ciências Contábeis. Logo, o conjunto de arquivos que atendiam a esse requisito foi considerado a população-alvo;

e) Dessa população-alvo foram feitas as análises dos dados.

A pesquisa se limitou a observar apenas a utilização das dissertações como construto teórico dos artigos, para melhor responder à problemática e ao objetivo, não considerando a utilização de teses nos artigos. $O$ tratamento dos dados foi realizado por meio da utilização do programa Microsoft Excel 2007®.

REUNIR - Revista de Administração, Contabilidade e Sustentabilidade - Vol. 2, nº 1, p.39-55, Jan-Abr/2012. 
Ressalta-se que algumas dissertações foram referenciadas com informações incompletas, como, por exemplo, com ausência do nome do programa e do curso, apresentando apenas o nome da instituição. Nesses casos, procurou-se nos sítios dos PPGCCs, com o auxílio da ferramenta de busca, a localização dessas dissertações pelos títulos e origem institucional. Persistindo ainda a impossibilidade de localização, tais dissertações foram dispostas em um conjunto denominado: Não encontrados. Desconsideraram-se as dissertações de anos anteriores a 1990, pois, além de serem em número pouco significante, eram de difícil acesso e localização.

\section{2 Áreas temáticas dos congressos pesquisadas}

Pelo fato de o EnANPAD tratar-se de um evento multidisciplinar, seccionado por áreas (algumas permanentes e outras de caráter temporário), a pesquisa focou-se em determinadas áreas temáticas de caráter mais próximo com a contabilidade, sendo elas: contabilidade, ensino e pesquisa em administração e contabilidade, finanças, gestão de operações e logística.

No caso do Congresso USP e do ANPCont, foram pesquisadas todas as áreas temáticas do evento, pois se assemelham de maneira geral, exceto pela área específica de Atuária, no Congresso USP. O Quadro 1 evidencia as áreas temáticas verificadas dos três congressos.

\begin{tabular}{|c|c|c|c|c|c|}
\hline Congressos & \multicolumn{5}{|c|}{ Áreas Temáticas Verificadas } \\
\hline ANPCont & $\begin{array}{c}\text { Controladoria e } \\
\text { Contabilidade } \\
\text { Gerencial }\end{array}$ & $\begin{array}{c}\text { Contabilidade para } \\
\text { Usuários Externos }\end{array}$ & $\begin{array}{c}\text { Mercados Financeiro, } \\
\text { de Crédito e de } \\
\text { Capitais }\end{array}$ & $\begin{array}{c}\text { Educação e } \\
\text { Pesquisa em } \\
\text { Contabilidade }\end{array}$ & - \\
\hline USP & $\begin{array}{c}\text { Controladoria e } \\
\text { Contabilidade } \\
\text { Gerencial }\end{array}$ & $\begin{array}{c}\text { Contabilidade para } \\
\text { Usuários Externos }\end{array}$ & $\begin{array}{c}\text { Mercados Financeiro, } \\
\text { de Crédito e de } \\
\text { Capitais }\end{array}$ & $\begin{array}{c}\text { Educação e } \\
\text { Pesquisa em } \\
\text { Contabilidade }\end{array}$ & ATUÁRIA \\
\hline EnANPAD & Contabilidade & $\begin{array}{c}\text { Gestão de Operações } \\
\text { e Logística }\end{array}$ & Finanças & $\begin{array}{c}\text { Ensino e Pesquisa } \\
\text { em Administração } \\
\text { e Contabilidade }\end{array}$ & - \\
\hline
\end{tabular}

Quadro 1 - Áreas temáticas analisadas dos três congressos

Fonte: Pesquisa (2010)

\subsection{Variáveis utilizadas em relação aos PPGCCs}

Como forma de parametrização sobre áreas temáticas das dissertações, utilizou-se a classificação dada por Lucena, Fernandes e Cavalcante (2009), que decidiram por bem estabelecer oito blocos de atuação, sendo eles:

- Usuários Externos (UE) - práticas, princípios, demonstrações contábeis etc.

- Usuários Internos (UI) - contabilidade gerencial, controladoria, gestão, orçamento, avaliação de desempenho, BSC, governança, custos etc.

- Educação, Ensino e Pesquisa em Contabilidade (EC) - currículo, metodologias empregadas, visão contabilística etc.

- Auditoria e Contabilidade Pública (AC) - orçamento, gestão, contabilidade pública, normas e princípios, auditoria interna e externa, perícia etc.

- Terceiro Setor (TS) - organizações não governamentais, responsabilidade social, ética contábil, contabilidade ambiental, capital intelectual etc.

REUNIR - Revista de Administração, Contabilidade e Sustentabilidade - Vol. 2, no 1, p.39-55, Jan-Abr/2012. 
- Tópicos Contemporâneos em Contabilidade (TC) - assuntos emergentes tratados na contabilidade, como, por exemplo, tecnologia da informação, economia, contabilidade comportamental, dentre outros.

- Atuária - cálculos atuariais, demografia, seguradoras, previdência etc.

- Mercado Financeiro e de Capitais (MFC) - finanças corporativas, avaliação de empresas, gestão de risco etc.

Essa classificação foi realizada pelos autores supracitados, mediante visita no sítio dos programas de contabilidade, com objetivo de classificá-los em áreas que não prejudicassem qualquer programa, tendo em vista a diversidade de cada programa quanto à temática estabelecida.

\section{APRESENTAÇÃO E ANÁLISE DOS RESULTADOS}

Para uma melhor visualização optou-se por desmembrar em quatro subtítulos a apresentação e a análise dos resultados.

\section{1 Área temática do evento $X$ Quantidade de artigos que utilizaram dissertações por ano}

Do total de 1.292 artigos dos eventos ANPCont, USP e EnANPAD, 201 artigos analisados corresponde ao evento ANPCont, sendo 47, 47 e 107 nos anos de 2007, 2008 e 2009, respectivamente. Dos artigos selecionados, 38\% utilizaram-se de dissertações na construção do trabalho, sendo 24 artigos em 2007, 19 em 2008 e 34 em 2009, num total de 77, havendo uma oscilação desse uso, mas com aumento no ano de 2009.

Observa-se que os artigos da área temática de controladoria e contabilidade gerencial utilizaram, em média, 41,7\% de dissertações como referência em 2007, passando pela área de contabilidade para usuários externos com 42,1\% em 2008 e 26,5\% em 2009, e a área de mercado financeiro, de créditos e de capitais até ensino e pesquisa em contabilidade com $26 \%$ em 2009. Nota-se uma certa homogeneidade na distribuição dos artigos entre as áreas temáticas do ANPCont em 2009.

Tabela 1 - Área temática do evento ANPCont x Quantidade de artigos que utilizaram dissertações por ano

\begin{tabular}{lcccccc}
\hline \multicolumn{1}{c}{ Área Temática - ANPCont } & \multicolumn{2}{c}{$\mathbf{2 0 0 7}$} & \multicolumn{2}{c}{$\mathbf{2 0 0 8}$} & \multicolumn{2}{c}{$\mathbf{2 0 0 9}$} \\
\cline { 2 - 7 } Controladoria e Contabilidade Gerencial & Qtde.* & $\mathbf{\%}$ & Qtde.* & $\mathbf{\%}$ & Qtde.* & $\mathbf{\%}$ \\
Contabilidade para Usuários Externos & 10 & 41,7 & 3 & 15,8 & 8 & 23,5 \\
Mercado Financeiro, de Créditos e de Capitais & 9 & 37,5 & 8 & 42,1 & 9 & 26,5 \\
Ensino e Pesquisa em Contabilidade & 1 & 4,2 & 3 & 15,8 & 8 & 23,5 \\
\hline TOTAL & 4 & 16,7 & 5 & 26,3 & 9 & 26,5 \\
\hline
\end{tabular}

* Quantidade de artigos que usam dissertações

Fonte: Pesquisa (2010)

Contudo, no ano de 2007, não foi possível analisar 1 artigo da área de contabilidade para usuários externos e, no ano de 2009, 11 artigos, sendo 3 artigos na área de controladoria e contabilidade gerencial, 4 na área de usuários externos e 4 na temática de ensino e pesquisa em contabilidade, por estarem indisponíveis no sítio do evento.

REUNIR - Revista de Administração, Contabilidade e Sustentabilidade - Vol. 2, no 1, p.39-55, Jan-Abr/2012. 
Já no evento USP, foram analisados 272 artigos científicos nas áreas temáticas. Deste total, 120 artigos correspondem ao ano de 2007, 84 ao ano de 2008 e 68 a 2009. Contudo, apenas 110 artigos (44\%) utilizaram dissertações dos programas de mestrados como fundamentação na construção dos artigos deste evento, sendo 56 no ano de 2007, 26 em 2008 e 28 em 2009, sendo perceptível uma queda no número de artigos que utilizaram dissertações entre os anos de 2007 a 2009.

A Tabela 2 especifica claramente as áreas temáticas do evento USP com os devidos percentuais de artigos que utilizaram dissertações como fonte de pesquisa. A área temática de contabilidade para usuários externos representa $37,5 \%$ dos artigos que referenciaram dissertações em 2007, a área de mercado financeiro, de créditos e de capitais obteve um percentual de $38,5 \%$ em 2008 , e a área de controladoria e contabilidade gerencial representou $46,4 \%$ em 2009.

Tabela 2 - Área temática do evento USP x Quantidade de artigos que utilizaram dissertações por ano

\begin{tabular}{lcccccc}
\hline \multicolumn{1}{c}{ Área Temática - USP } & \multicolumn{2}{c}{$\mathbf{2 0 0 7}$} & \multicolumn{2}{c}{$\mathbf{2 0 0 8}$} & \multicolumn{2}{c}{$\mathbf{2 0 0 9}$} \\
\cline { 2 - 7 } Controladoria e Contabilidade Gerencial & Qtde.* & $\mathbf{\%}$ & Qtde.* & $\mathbf{\%}$ & Qtde.* & $\mathbf{\%}$ \\
Contabilidade para Usuários Externos & 14 & 25,0 & 7 & 26,9 & 13 & 46,4 \\
Mercados Financeiro, de Crédito e de Capitais & 21 & 37,5 & 6 & 23,1 & 10 & 35,7 \\
Educação e Pesquisa em Contabilidade & 11 & 19,6 & 10 & 38,5 & 0 & 0,0 \\
Atuária & 8 & 14,3 & 1 & 3,8 & 4 & 14,3 \\
TOTAL & 2 & 3,6 & 2 & 7,7 & 1 & 3,6 \\
\hline
\end{tabular}

* Quantidade de artigos que usam dissertações

Fonte: Pesquisa (2010)

No congresso EnANPAD o total de artigos em 2007 foi de 301, em 2008 havia 284 e em 2009 encontravam-se 234 artigos, totalizando dessa forma 819 artigos verificados. Desses, apenas 293 artigos utilizaram dissertações (de um modo geral) no processo de desenvolvimento do estudo, havendo uma queda no uso de dissertações no decorrer dos anos, sendo 120 artigos em 2007, 91 em 2008 e 82 em 2009. A Tabela 3 detalha essa distribuição de acordo com a área temática do evento EnANPAD.

Tabela 3 - Área temática do evento EnANPAD x Quantidade de artigos que utilizaram dissertações por ano

\begin{tabular}{lcccccc}
\hline \multicolumn{1}{c}{ Área temática - EnANPAD } & \multicolumn{2}{c}{$\mathbf{2 0 0 7}$} & \multicolumn{2}{c}{$\mathbf{2 0 0 8}$} & \multicolumn{2}{c}{$\mathbf{2 0 0 9}$} \\
\cline { 2 - 7 } Contabilidade & Qtde.* & $\mathbf{\%}$ & Qtde.* & $\mathbf{\%}$ & Qtde.* & $\mathbf{\%}$ \\
Ensino e Pesquisa em Administração e Contabilidade & 51 & 42,5 & 35 & 38,5 & 32 & 39,0 \\
Finanças & 29 & 24,2 & 27 & 29,7 & 17 & 20,7 \\
Gestão de Operações e Logística & 23 & 19,2 & 20 & 22,0 & 25 & 30,5 \\
TOTAL & 17 & 14,2 & 9 & 9,9 & 8 & 9,8 \\
\hline
\end{tabular}

* Quantidade de artigos que usam dissertações

Fonte: Pesquisa (2010)

Observa-se que os artigos alocados na área temática contabilidade utilizam mais dissertações contemplando, em média, $40 \%$ do total destas, em relação às demais áreas analisadas.

REUNIR - Revista de Administração, Contabilidade e Sustentabilidade - Vol. 2, no 1, p.39-55, Jan-Abr/2012. 


\subsection{Dissertações referenciadas nos artigos por cursos}

A Tabela 4 evidencia as frequências com que os artigos utilizaram dissertações como fonte de pesquisa e os cursos de pós-graduação que mais tiveram suas dissertações referenciadas. As dissertações utilizadas pelos artigos foram dispostas por curso, para obter maior conhecimento daqueles que contribuem na elaboração desses artigos, já que as áreas temáticas estudadas nos três eventos são referentes à contabilidade e pretende-se inferir alguns resultados com essa informação.

Ressalta-se que o número de dissertações dispostas na Tabela 4 não corresponde necessariamente a distintas dissertações, pois um mesmo artigo pode conter diversas referências de dissertações (houve casos em que um artigo possuía 11 referências desse tipo de pesquisa, por exemplo), além de dois ou mais distintos artigos coincidirem na utilização de uma mesma dissertação.

Para evidenciar a frequência dos cursos de pós-graduação, foram considerados os que se apresentavam com maior frequência e as que possuem relação mais direta, como áreas afins e semelhantes à área contábil, e os demais cursos de pós-graduação foram dispostos em uma única frequência chamada de "outros", conforme mostra a Tabela 4. Seguindo a ideia de Richardson (2009) os "outros" cursos não apresentam número significativo de utilização nos artigos dos eventos estudados, sendo mais viável o agrupamento dessas informações, dado que estas não impedem a verificação e a análise dos resultados como um todo.

Tabela 4 - Dissertações referenciados nos artigos por cursos

\begin{tabular}{|c|c|c|c|c|c|c|c|c|c|}
\hline \multirow{2}{*}{ Curso } & \multicolumn{3}{|c|}{ ANPCont } & \multicolumn{3}{|c|}{ USP } & \multicolumn{3}{|c|}{ EnANPAD } \\
\hline & 2007 & 2008 & 2009 & 2007 & 2008 & 2009 & 2007 & 2008 & 2009 \\
\hline Contabilidade & 17 & 20 & 43 & 53 & 31 & 19 & 63 & 56 & 53 \\
\hline Administração & 9 & 10 & 20 & 13 & 12 & 9 & 47 & 58 & 78 \\
\hline Engenharia de Produção & 10 & 2 & 4 & 11 & 3 & 9 & 21 & 29 & 20 \\
\hline Economia & 3 & 4 & 2 & 4 & 2 & 3 & 5 & 11 & 13 \\
\hline Outros & 2 & 3 & 4 & 2 & 2 & 11 & 18 & 16 & 23 \\
\hline Não encontrados & 0 & 0 & 0 & 4 & 3 & 0 & 5 & 5 & 4 \\
\hline
\end{tabular}

Fonte: Pesquisa (2010)

Embora o foco desta pesquisa seja apenas o curso de contabilidade, observa-se uma participação significativa das dissertações dos cursos de administração e engenharia de produção. Nota-se que o uso das dissertações do curso de administração foi mais frequente do que contabilidade em 2008 e 2009 no evento EnANPAD, possivelmente pela característica do evento, ou seja, ser este um evento de administração e contabilidade. Nos demais eventos, ANPCont e USP, há um equilíbrio entre as áreas de administração e engenharia de produção.

\section{3 Área Temática X Dissertações utilizadas por PPGCCs}

A seguir, as Tabelas 5, 6 e 7 apresentam os PPGCCs que mais se destacaram (em negrito) por terem as suas dissertações referenciadas nos artigos dos eventos analisados por cada área temática dos eventos.

REUNIR - Revista de Administração, Contabilidade e Sustentabilidade - Vol. 2, no 1, p.39-55, Jan-Abr/2012. 
Tabela 5 - Área Temática - ANPCont x Dissertações utilizadas por PPGCCs

\begin{tabular}{|c|c|c|c|c|}
\hline ÁREAS TEMÁTICAS - ANPCont & PPGCC & 2007 & 2008 & 2009 \\
\hline \multirow{8}{*}{$\begin{array}{c}\text { Controladoria e } \\
\text { Contabilidade Gerencial }\end{array}$} & UFRJ & 0 & 0 & 1 \\
\hline & UERJ & 1 & 0 & 0 \\
\hline & USP & 5 & $\mathbf{0}$ & 6 \\
\hline & UNIFECAP & 0 & 1 & 0 \\
\hline & UMESP & 1 & 0 & 0 \\
\hline & UNISINOS & 0 & 0 & 1 \\
\hline & FURB & 1 & 1 & 0 \\
\hline & UNB/UFPB/UFRN & 1 & 0 & 1 \\
\hline \multirow{5}{*}{$\begin{array}{l}\text { Contabilidade para } \\
\text { Usuários Externos }\end{array}$} & FUCAPE & $\mathbf{0}$ & 4 & 3 \\
\hline & UERJ & 1 & 0 & 0 \\
\hline & USP & 1 & 3 & 3 \\
\hline & UNIFECAP & 0 & 0 & 2 \\
\hline & UNB/UFPB/UFRN & 3 & 1 & 1 \\
\hline \multirow{4}{*}{$\begin{array}{l}\text { Mercados Financeiro, } \\
\text { de Crédito e de Capitais }\end{array}$} & FUCAPE & $\mathbf{0}$ & 1 & 4 \\
\hline & USP & 0 & 3 & 0 \\
\hline & UNIFECAP & 0 & 0 & 1 \\
\hline & UNB/UFPB/UFRN & 0 & 1 & 0 \\
\hline \multirow{7}{*}{$\begin{array}{l}\text { Educação e Pesquisa } \\
\text { em Contabilidade }\end{array}$} & UFRJ & 0 & 0 & 1 \\
\hline & USP & 2 & 4 & 2 \\
\hline & UNIFECAP & $\mathbf{0}$ & 1 & 12 \\
\hline & PUCSP & 0 & 0 & 2 \\
\hline & UFSC & 1 & 0 & 0 \\
\hline & FVC - BA & 0 & 0 & 1 \\
\hline & UNB/UFPB/UFRN & 0 & 0 & 2 \\
\hline \multicolumn{2}{|c|}{ TOTAL DE DISSERTAÇÕES DOS PPGCCs } & 17 & 20 & 43 \\
\hline
\end{tabular}

Fonte: Pesquisa (2010)

A Tabela 5 apresenta os PPGCCs que mais tiveram suas dissertações referenciadas nos artigos do evento ANPCont. Dentre eles, evidencia-se o PPGCC da USP nos anos de 2007 e 2009 na área temática de controladoria e contabilidade gerencial. A Fucape e a USP, nos anos de 2008 e 2009, aparecem em todas as áreas temáticas abordadas, sendo que a USP destaca-se na área controladoria e contabilidade gerencial e a Fucape nas áreas de contabilidade para usuários externos e mercados financeiro, de crédito e de capitais. Por fim, na área de educação e pesquisa em contabilidade, a UniFecap se sobressai por ter suas dissertações mais referenciadas nesta temática no ano 2009.

Quanto às variáveis utilizadas em relação aos PPGCCs (descritas no item 3.3), as dissertações analisadas nos artigos foram classificadas, na sua maioria, nas áreas de usuários internos, no ano de 2007, na área de educação, ensino e pesquisa em contabilidade e de mercado financeiro e de capitais, para o ano de 2008, e no ano de 2009 a ênfase é dada à área de educação, ensino e pesquisa em contabilidade e auditoria e contabilidade pública.

A Tabela 6 apresenta o número de dissertações dos PPGCCs nos artigos do evento USP. As dissertações do PPGCC da USP se sobressaem, sendo referenciadas em quase todas as áreas temáticas do evento nos anos de 2007 a 2009, como mostra a Tabela 6, com exceção da área de educação e pesquisa em contabilidade que tem como destaque as dissertações, mais uma vez, da UniFecap.

REUNIR - Revista de Administração, Contabilidade e Sustentabilidade - Vol. 2, no 1, p.39-55, Jan-Abr/2012. 
Tabela 6 - Área Temática - USP x Dissertações utilizadas por PPGCCs

\begin{tabular}{|c|c|c|c|c|}
\hline ÁREAS TEMÁTICAS - USP & PPGCC & 2007 & 2008 & 2009 \\
\hline \multirow{8}{*}{$\begin{array}{c}\text { Controladoria e } \\
\text { Contabilidade Gerencial }\end{array}$} & UFRJ & 1 & 0 & 0 \\
\hline & USP & 8 & 3 & 2 \\
\hline & CEPPEV & 1 & 0 & 0 \\
\hline & UNIFECAP & 1 & 0 & 0 \\
\hline & PUCSP & 1 & 0 & 0 \\
\hline & UNISINOS & 0 & 1 & 3 \\
\hline & FURB & 2 & 0 & 0 \\
\hline & UNB/UFPB/UFRN & 1 & 2 & 2 \\
\hline \multirow{8}{*}{$\begin{array}{l}\text { Contabilidade para } \\
\text { Usuários Externos }\end{array}$} & FUCAPE & 2 & 0 & 7 \\
\hline & USP & 11 & 5 & $\mathbf{0}$ \\
\hline & UNIFECAP & 2 & 0 & 0 \\
\hline & PUCSP & 1 & 0 & 0 \\
\hline & UNISINOS & 0 & 1 & 1 \\
\hline & FURB & 1 & 2 & 0 \\
\hline & UFSC & 1 & 0 & 0 \\
\hline & UNB/UFPB/UFRN & 3 & 1 & 0 \\
\hline \multirow{4}{*}{$\begin{array}{l}\text { Mercados Financeiro, } \\
\text { de Crédito e de Capitais }\end{array}$} & FUCAPE & 0 & 4 & 0 \\
\hline & UFRJ & 1 & 0 & 0 \\
\hline & USP & 10 & 2 & $\mathbf{0}$ \\
\hline & UNB/UFPB/UFRN & 1 & 1 & 0 \\
\hline \multirow{5}{*}{$\begin{array}{l}\text { Educação e Pesquisa } \\
\text { em Contabilidade }\end{array}$} & FURB & 0 & 0 & 1 \\
\hline & USP & 0 & 1 & 2 \\
\hline & UNIFECAP & 2 & 4 & $\mathbf{0}$ \\
\hline & UNISINOS & 0 & 0 & 0 \\
\hline & UNB/UFPB/UFRN & 0 & 0 & 1 \\
\hline \multirow{2}{*}{ Atuária } & USP & 3 & 2 & $\mathbf{0}$ \\
\hline & UNB/UFPB/UFRN & 0 & 2 & 0 \\
\hline \multicolumn{2}{|c|}{ TOTAL DE DISSERTAÇÕES DOS PPGCCs } & 53 & 31 & 19 \\
\hline
\end{tabular}

Fonte: Pesquisa (2010)

Note-se, a título de exemplo, que na Tabela 6, embora a Tabela 2 tenha apresentado um trabalho em 2009 na área de Atuária, com dissertação citada na referência, essa dissertação não faz parte de nenhum PPGCC, podendo ser de programas de outros cursos. Assim, foram contabilizados nas Tabelas 5, 6 e 7, apenas as dissertações de programas de Contabilidade. Nas tabelas 1, 2 e 3, por sua vez, foram contabilizados em número absoluto os artigos que simplesmente apresentaram dissertações nas referências.

Quanto à classificação das variáveis observadas e seguindo a classificação dada por Lucena, Fernandes e Cavalcante (2009), no ano de 2007 as dissertações referenciadas nos artigos contemplam a temática de usuários externos e mercado financeiro e de capitais. Em 2008 notam-se as dissertações referenciadas na temática de usuários externos e usuários internos, e também a de mercado financeiro e de capitais. Por fim, o ano de 2009 ressalta as dissertações com temática para usuários internos e educação, ensino e pesquisa em contabilidade.

O Tabela 7 apresenta o PPGCC da USP e da UnB/UFPB/UFRN como os mais frequentes no evento EnANPAD, ou seja, as dissertações mais utilizadas concentram-se nestes programas de pós-graduação.

REUNIR - Revista de Administração, Contabilidade e Sustentabilidade - Vol. 2, no 1, p.39-55, Jan-Abr/2012. 
Tabela 7 - Área Temática - EnANPAD x Dissertações utilizadas por PPGCCs

\begin{tabular}{|c|c|c|c|c|}
\hline ÁREAS TEMÁTICAS - EnANPAD & PPGCC & 2007 & 2008 & 2009 \\
\hline \multirow{14}{*}{ Contabilidade } & FUCAPE & 4 & 7 & 3 \\
\hline & PUCRJ & 1 & 0 & 0 \\
\hline & UFRJ & 3 & 1 & 3 \\
\hline & UERJ & 1 & 0 & 2 \\
\hline & USP & 20 & 15 & 18 \\
\hline & USPRP & 0 & 0 & 1 \\
\hline & UPM & 0 & 1 & 0 \\
\hline & UNIFECAP & 1 & 3 & 2 \\
\hline & UNISINOS & 1 & 0 & 4 \\
\hline & FURB & 1 & 0 & 2 \\
\hline & UFPR & 0 & 0 & 1 \\
\hline & UFSC & 0 & 1 & 0 \\
\hline & UNB/UFPB/UFRN & 9 & 4 & 5 \\
\hline & CEPPEV & 1 & 0 & 0 \\
\hline \multirow{6}{*}{$\begin{array}{c}\text { Ensino e Pesquisa em } \\
\text { Administração e Contabilidade }\end{array}$} & FUCAPE & 0 & 1 & 0 \\
\hline & UFRJ & 0 & 1 & 0 \\
\hline & USP & 5 & 6 & 6 \\
\hline & UNIFECAP & 0 & 6 & 0 \\
\hline & UNISINOS & 1 & 0 & 0 \\
\hline & UNB/UFPB/UFRN & 13 & 4 & 2 \\
\hline \multirow{7}{*}{ Finanças } & FUCAPE & 0 & 2 & 1 \\
\hline & UFRJ & 0 & 1 & 0 \\
\hline & UERJ & 0 & 1 & 0 \\
\hline & USP & 2 & $\mathbf{0}$ & 2 \\
\hline & USPRP & 0 & 0 & 1 \\
\hline & UNIFECAP & 0 & 1 & 0 \\
\hline & UNB/UFPB/UFRN & 0 & 1 & 0 \\
\hline \multicolumn{2}{|c|}{ Gestão de Operações e Logística } & \multicolumn{3}{|c|}{ Não houve } \\
\hline \multicolumn{2}{|c|}{ TOTAL DE DISSERTAÇÕES DOS PPGCCS } & 63 & 56 & 53 \\
\hline
\end{tabular}

Fonte: Pesquisa (2010)

Constata-se que, no EnANPAD, a área temática contabilidade revela as dissertações da USP com maior frequência durante os anos e, de acordo com a classificação de Lucena, Fernandes e Cavalcante (2009), a área mais frequente dessas dissertações refere-se a usuários internos, sendo utilizadas, pelos artigos, 7 dissertações em 2007, 5 em 2008 e 14 em 2009. A UnB/UFPB/UFRN, com maior frequência na área temática do evento: ensino e pesquisa em administração e contabilidade apresentam dissertações mais comuns nessa mesma área temática do evento.

É relevante enfatizar a ausência de dissertações na área temática gestão de operações e logística. Tal fato pode ser explicado pela pouca ou provável inexistência de dissertações nos PPGCCs sobre a temática, podendo ser este um amplo campo de estudo a ser explorado pelos docentes e discentes dos PPGCCs de todo o país. Ressalta-se que as dissertações utilizadas pelos artigos (conforme Tabela 3) dentro dessa temática foram, em sua maioria, dos cursos de Pós-Graduação Stricto Sensu de Engenharia de Produção e, em seguida, de Administração.

\subsection{Ano de publicação das dissertações utilizadas}

A utilização de materiais a serem referenciados nos artigos deve procurar ser sempre atualizada ou recente, pelo menos, essa é a visão de avaliadores dos diversos eventos na área

REUNIR - Revista de Administração, Contabilidade e Sustentabilidade - Vol. 2, no 1, p.39-55, Jan-Abr/2012. 
contábil. Isso pode ser percebido pelos critérios de avaliação que são disponibilizados pelos sítios desses eventos. Nesse sentido, observou-se que houve uma participação significativa de dissertações antigas nos artigos dos congressos estudados, conforme evidencia a Tabela 8.

Para um melhor entendimento, consideram-se, nesta pesquisa, dissertações recentes as utilizadas nos artigos a partir de 2004 (em negrito), pois abrangem os três anos dos eventos analisados.

Tabela 8 - Ano de publicação das dissertações utilizadas

\begin{tabular}{c|c|c|c|c|c|c|c|c|c}
\hline \multirow{2}{*}{ Ano da Dissertação } & \multicolumn{3}{|c|}{ ANPCont } & \multicolumn{4}{c}{ USP } & \multicolumn{3}{c}{ EnANPAD } \\
\cline { 2 - 11 } & $\mathbf{2 0 0 7}$ & $\mathbf{2 0 0 8}$ & $\mathbf{2 0 0 9}$ & $\mathbf{2 0 0 7}$ & $\mathbf{2 0 0 8}$ & $\mathbf{2 0 0 9}$ & $\mathbf{2 0 0 7}$ & $\mathbf{2 0 0 8}$ & $\mathbf{2 0 0 9}$ \\
\hline 1990 a 1998 & 7 & 1 & 3 & 10 & 3 & 1 & 5 & 4 & 1 \\
\hline 1999 & 0 & 0 & 1 & 0 & 0 & 0 & 1 & 2 & 1 \\
\hline 2000 & 1 & 0 & 3 & 4 & 1 & 1 & 2 & 4 & 2 \\
\hline 2001 & 2 & 1 & 1 & 1 & 1 & 0 & 3 & 2 & 3 \\
\hline 2002 & 0 & 1 & 6 & 6 & 3 & 1 & 7 & 4 & 3 \\
\hline 2003 & 3 & 1 & 5 & 8 & 4 & 2 & 14 & 8 & 7 \\
\hline $\mathbf{2 0 0 4}$ & $\mathbf{1}$ & $\mathbf{7}$ & $\mathbf{6}$ & $\mathbf{1 6}$ & $\mathbf{5}$ & $\mathbf{5}$ & $\mathbf{1 3}$ & $\mathbf{1 0}$ & $\mathbf{1 1}$ \\
\hline $\mathbf{2 0 0 5}$ & $\mathbf{3}$ & $\mathbf{2}$ & $\mathbf{4}$ & $\mathbf{5}$ & $\mathbf{8}$ & $\mathbf{0}$ & $\mathbf{8}$ & $\mathbf{9}$ & $\mathbf{7}$ \\
\hline $\mathbf{2 0 0 6}$ & $\mathbf{0}$ & $\mathbf{5}$ & $\mathbf{8}$ & $\mathbf{3}$ & $\mathbf{5}$ & $\mathbf{3}$ & $\mathbf{9}$ & $\mathbf{9}$ & $\mathbf{8}$ \\
\hline $\mathbf{2 0 0 7}$ & $\mathbf{0}$ & $\mathbf{2}$ & $\mathbf{4}$ & $\mathbf{0}$ & $\mathbf{1}$ & $\mathbf{5}$ & $\mathbf{1}$ & $\mathbf{3}$ & $\mathbf{5}$ \\
\hline $\mathbf{2 0 0 8}$ & $\mathbf{0}$ & $\mathbf{0}$ & $\mathbf{2}$ & $\mathbf{0}$ & $\mathbf{0}$ & $\mathbf{1}$ & $\mathbf{0}$ & $\mathbf{1}$ & $\mathbf{5}$ \\
\hline TOTAL DE DISSERTAÇÕES & $\mathbf{1 7}$ & $\mathbf{2 0}$ & $\mathbf{4 3}$ & $\mathbf{5 3}$ & $\mathbf{3 1}$ & $\mathbf{1 9}$ & $\mathbf{6 3}$ & $\mathbf{5 6}$ & $\mathbf{5 3}$ \\
\hline
\end{tabular}

Fonte: Pesquisa (2010)

Nos três eventos, constata-se uma expressiva utilização de dissertações publicadas em 2004. No entanto, apesar de ser um dos critérios de avaliação a questão da utilização de pesquisas recentes no processo de construção de artigos (como é o caso em estudo), observase que há uma utilização de dissertações não tão recentes.

Nos eventos analisados, em 2007, as dissertações anteriores a 2004 chegam a representar 50\% ou mais, mas em 2008 e 2009 esse percentual reduz, aumentando a utilização de dissertações recentes.

\section{CONSIDERAÇÕES FINAIS}

Diante da clara evidência de as dissertações dos programas de mestrados serem uma fonte de pesquisa robusta e de qualidade para o desenvolvimento de artigos científicos na área, o presente estudo buscou evidenciar a o perfil da utilização dos mesmos nos eventos ANPCont, EnANPAD e USP no período de 2007 a 2009.

No evento ANPCont observou-se uma oscilação, durante os anos, da quantidade de artigos que utilizaram dissertações, mas houve um considerável aumento de $33 \%$ da utilização de dissertações de PPGCCs, com destaque para os programas da USP na área temática do evento: controladoria e contabilidade gerencial e contabilidade para usuários externos. Destaque, também, para a Fucape nesta última área citada e na de mercados financeiro, de créditos e de capitais; a UniFecap se sobressaiu na área de educação e pesquisa em contabilidade em 2009, com 12 dissertações sendo referenciadas.

Constatou-se que os artigos da área temática do ANPCont que mais utilizaram dissertações foi a de contabilidade para usuários externos e que houve um relevante aumento do uso de dissertações mais recentes.

No evento USP, constatou-se sistemática queda no uso de dissertações referenciadas pelos artigos e de dissertações de PPGCCs. Ainda assim, o PPGCC mais destacado é o da

REUNIR - Revista de Administração, Contabilidade e Sustentabilidade - Vol. 2, no 1, p.39-55, Jan-Abr/2012. 
própria USP, exceto na área temática sobre educação e pesquisa em contabilidade, onde a UniFecap, mais uma vez, se sobressaiu dentre os demais programas. Constatou-se, novamente, que a área temática da USP que mais utilizou dissertações foi a de contabilidade para usuários externos, e houve queda no uso de dissertações mais recentes.

Observa-se, pelos resultados, uma uniformidade nas áreas contabilidade para usuários externos no que se remete à utilização mais assídua de dissertações. Tal fato sugere que há uma gama de dissertações nessa área, facilitando, assim, a produção de mais pesquisas. No entanto, há lacunas em outras áreas, como na área de mercado financeiro, gestão de operações e logística, as quais precisam ser mais exploradas dentro dos programas em contabilidade.

No evento EnANPAD também se constatou sistemática queda no uso de dissertações referenciadas pelos artigos e de dissertações de PPGCCs. O PPGCC mais destacado é o da USP, exceto na área temática sobre educação e pesquisa em contabilidade, onde as dissertações da UNB/UFPB/UFRN obtiveram maior utilização por parte dos artigos desse evento. Os artigos da área temática contabilidade foram os que mais utilizaram dissertações, havendo aumento dessas pesquisas mais recentes.

Nos três eventos é possível observar que o PPGCC da USP teve suas dissertações referenciadas em maior quantidade em relação aos demais programas. Tal fato também foi verificado por Gallon et al. (2008), pois, em seu estudo epistemológico, identificaram que, independentemente da perspectiva analisada, as teses e dissertações da USP foram as mais empregadas como referência. Embora o resultado das autoras tenha sido generalizado a toda a USP, fica observado neste estudo que o mesmo se aplica ao PPGCC, e isso pode ser justificado pelo tempo de existência de tal programa.

Posteriormente nota-se a presença das dissertações desenvolvidas pelos programas da UniFecap, Fucape e UNB/UFPB/UFRN, devendo-se levar em consideração que, neste último programa mencionado, por ser considerado multiinstitucional, as dissertações referenciadas no período anterior a 2007 devem contemplar o curso de mestrado da UFPE, por fazer parte do grupo até esse período.

As áreas dos PPGCCs mais utilizadas pelos artigos, conforme classificação dada por Lucena, Fernandes e Cavalcante (2009), versam entre: usuários internos, educação, ensino e pesquisa em contabilidade, usuários externos e mercado financeiro e de capitais, sugerindo uma necessidade de pesquisas nas demais áreas estabelecidas por esses autores.

Contudo, observa-se, nos eventos USP e EnANPAD, queda na utilização de dissertações de áreas afins à contabilidade no construto de artigos em contabilidade, mesmo com um número crescente de PPGCCs no Brasil. Deve-se considerar, também, o tempo de existência desses programas e a consequente evolução no número de dissertações elaboradas nesses programas que podem ser utilizadas como fonte de pesquisa científica devido à sua robustez de conteúdo.

Esta pesquisa evidencia, entre outras considerações, potenciais campos de estudos, mas sugere-se que novas pesquisas continuem essa observância em áreas determinadas, ou com a utilização de periódicos, por exemplo, para corroborar, ou não, os resultados obtidos ou abrir novas discussões.

\section{REFERÊNCIAS}

CHAN, Betty L.; MILANI FILHO, Marco A. F.; MARTINS, Gilberto de A. Utilização da Análise de Correspondência Para Uma Abordagem Bibliométrica: Relação Entre a Área

REUNIR - Revista de Administração, Contabilidade e Sustentabilidade - Vol. 2, no 1, p.39-55, Jan-Abr/2012. 
Temática e a Plataforma Teórica. In: Anais... XXXI ENCONTRO NACIONAL DOS PROGRAMAS DE PÓS-GRADUAÇÃO EM ADMINISTRAÇÃO, Rio de Janeiro/RJ, 2007.

DIEHL, Carlos A.; SOUZA, Marcos A. de; ALVES, Tiago W. Custeio Baseado em Atividades (ABC): Um Estudo Sobre Publicações em Eventos Científicos. In: Anais... XXXII ENCONTRO NACIONAL DOS PROGRAMAS DE PÓS-GRADUAÇÃO EM ADMINISTRAÇÃO, Rio de Janeiro/RJ, 2008.

FREZATTI, F; BORBA, J. Análise dos traços de tendência de uma amostra das revistas científicas da área de contabilidade publicadas em língua inglesa. Caderno de Estudos Fipecafi . v. 13, n. 25, p. 50-78, 2000.

GALlON, Alessandra V. et al. Estudo Epistemológico da Produção Científica sobre as Perspectivas Contábil e Econômica do Capital Intelectual Realizada no Brasil. In: Anais... XXXII ENCONTRO NACIONAL DOS PROGRAMAS DE PÓS-GRADUAÇÃO EM ADMINISTRAÇÃO, Rio de Janeiro/RJ, 2008.

GIL, Antonio C. Como elaborar projetos de pesquisa. 4ª Ed. São Paulo: Atlas, 2009.

GOMES, Carlos A. S. et al. Produção Científica em Contabilidade Pública: uma Análise dos Artigos Apresentados no EnANPAD e Congresso USP de Controladoria e Contabilidade no Período 2004-2007. In. Anais... XXXII ENCONTRO NACIONAL DOS PROGRAMAS DE PÓS-GRADUAÇÃO EM ADMINISTRAÇÃO, Rio de Janeiro/RJ, 2008.

GOMES, Rafael B. et al. Uma abordagem investigativa nas dissertações de mestrado do Programa Multiinstitucional em Ciências Contábeis sob a ótica de projeto de pesquisa. In: Anais... I Seminário UFPE de Ciências Contábeis, Recife/PE, 2007.

LUCENA, Wenner G. L.; CAVALCANTE, Paulo R. N.; SALES, Luciana B. O Perfil das Dissertações do Programa Multiintitucional e Inter-regional de Pós-Graduação em Ciências Contábeis UnB/UFPB/UFRN/UFPE. In: Anais... XXXI ENCONTRO NACIONAL DOS PROGRAMAS DE PÓS-GRADUAÇÃO EM ADMINISTRAÇÃO, Rio de Janeiro/RJ, 2007.

LUCENA, Wenner G. L. L.; FENANDES, Maria S. A. F.; CAVALCANTE, Paulo R. N. As Tendências, Desafios e Perspectivas da Pesquisa nos Programas de Pós-Graduação em Ciências Contábeis no Brasil: Um Estudo com Base nas Dissertações. In: Anais... XVI CONGRESSO BRASILEIRO DE CUSTOS - CBC, Fortaleza/CE, 2009.

MARTINS, Orleans S. Mestres em ciências contábeis pelo programa Multiinstitucional da UNB/UFPB/UFPE/UFRN: Uma análise a partir de suas percepções e avaliações. Dissertação (Mestrado em Ciências Contábeis) - Programa Multiinstitucional e InterRegional de Pós-Graduação em Ciências Contábeis, Universidade de Brasília, Universidade Federal da Paraíba e Universidade Federal do Rio Grande do Norte, João Pessoa/PB, 2009, p.126.

REUNIR - Revista de Administração, Contabilidade e Sustentabilidade - Vol. 2, nº 1, p.39-55, Jan-Abr/2012. 
MENDONÇA NETO, O.R.; RICCIO, E. L.; SAKATA, M.C.G. Paradigmas de Pesquisa em Contabilidade no Brasil: EnANPAD: 1981 - 2005. XXX ENCONTRO NACIONAL DOS PROGRAMAS DE PÓS-GRADUAÇÃO EM ADMINISTRAÇÃO, Salvador/BA, 2006.

NASCIMENTO, Artur R.; JUNQUEIRA, Emanuel; MARTINS, Gilberto de A. Análise Epistemológica da Produção Científica em Contabilidade Gerencial no Brasil. In: Anais... XXXIII ENCONTRO NACIONAL DOS PROGRAMAS DE PÓS-GRADUAÇÃO EM ADMINISTRAÇÃO, Rio de Janeiro/RJ, 2009.

RICCIO, Edson L.; SAKATA; Marici G.; CARASTAN; Jacira T. A Pesquisa Contábil nas Universidades Brasileiras [1962-1999]. Caderno de Estudos (FEA/USP), São Paulo, p. 3544, 1999.

RICHARDSON, Roberto J. Pesquisa social: métodos e técnicas. $3^{\text {a }}$ Ed. São Paulo: Atlas, 2009.

RUIZ, João A. Metodologia Científica: guia para eficiência nos estudos. $6^{a}$ Ed. São Paulo: Atlas, 2006.

SANTOS, Nálbia de A.; LIMA, Severino C. de; MARTINS, Gilberto de A. Análise do Referencial Bibliográfico de Dissertações do Programa Multiinstitucional de Pós-Graduação em Ciências Contábeis (UFPB, UFPE, UFRN E UNB). In: Anais... XXXIII ENCONTRO NACIONAL DOS PROGRAMAS DE PÓS-GRADUAÇÃO EM ADMINISTRAÇÃO, Rio de Janeiro/RJ, 2009.

SOUZA, Ivone G. de A. Uma análise das abordagens epistemológicas e metodológicas da pesquisa contábil no Programa do Mestrado Multiinstitucional em Ciências Contábeis. 2005. 1360f. Dissertação (Mestrado em Ciências Contábeis) - Programa Multiinstitucional e InterRegional de Pós-Graduação em Ciências Contábeis, Universidade de Brasília, Universidade Federal da Paraíba, Universidade Federal de Pernambuco e Universidade Federal do Rio Grande do Norte, Recife, 2005.

SPESSATTO, Giseli e BEZERRA, Francisco A. Utilização das abordagens positiva $e$ normativa nas pesquisas em contabilidade. In: Anais... XV CONGRESSO BRASILEIRO DE CUSTOS - CBC, 2008, Curitiba/PR, 2008.

THEÓFILO, Carlos R; IUDICIBUS, Sérgio de. Uma Análise Crítico-Epistemológica da Produção Científica em Contabilidade no Brasil. In: Anais... XXXIII ENCONTRO NACIONAL DOS PROGRAMAS DE PÓS-GRADUAÇÃO EM ADMINISTRAÇÃO Brasília/DF, 2005. 